\title{
Dual-Frequency Reflectarray Cell to Provide Opposite Phase Shift in Dual Circular Polarization With Application in Multibeam Satellite Antennas
}

\author{
Daniel Martinez-de-Rioja \\ Eduardo Carrasco
}

\author{
Rafael Florencio , Jose A. Encinar' \\ and Rafael R. Boix
}

\begin{abstract}
A new reflectarray cell is proposed to simultaneously provide opposite phase shifts between orthogonal circular polarizations at two frequencies $(19.7$ and $29.5 \mathrm{GHz}$ ) by applying a variable rotation technique independently at each frequency. The reflectarray cell, which consists of two dielectric layers with two levels of printed elements (dipoles and arcs), has been characterized for both circular and linear polarizations at each frequency. Apart from the implementation of the variable rotation technique at each frequency, the reflectarray cell provides an additional phase adjustment at the higher frequency, which can be used to shape the beam. This concept applied to multiple spot satellites in $K a$-band will allow to produce two adjacent beams per feed in orthogonal circular polarizations at transmission and reception frequency bands.
\end{abstract}

Index Terms-Dual-circular polarization (CP), Ka-band, multispot satellites, reflectarray, variable rotation technique (VR'T).

\section{INTRODUCTION}

$\mathbf{O}$ VER the past few years, reflectarrays have been proposed to reduce the number of antennas required on board the high-throughput satellites in $\mathrm{Ka}$-band [1] by generating two different beams per feed, through the use of reflectarray cells able to provide opposite phase shift in orthogonal polarizations on a parabolic reflectaray [2] or operate at different frequencies [3] using a flat reflectarray. In contrast to the currently used reflectors, reflectarrays can provide a different phase shift in each polarization by an appropriate design of the reflectarray cells, allowing to generate different beams, either in each linear [4]-[6] or circular [2], [7], [8] polarization.

While the design of reflectarray cells with independent phase control in linear polarization (LP) is straightforward, by using rectangular patches [4], [5], or orthogonal sets of dipoles for each LP [6], the design of reflectarray cells with independent control in each orthogonal circular polarization (CP) is more complex. Some reflectarray cells have been proposed to operate in dual CP with independent phase control in each CP [7], [8]. Both reflectarrays reported in [7] and [8] require voluminous multilayer configuration and operate on a single frequency band.

A parabolic reflectarray that generates two closely spaced beams in orthogonal $\mathrm{CP}$ with a feed operating in dual $\mathrm{CP}$ was proposed in [2]. The technique is grounded in a unique property of reflectarrays based on the variable rotation technique (VRT) [9]-[11], more explicitly, the phase shift of the reflected field in right-handed CP (RHCP) is proportional to twice the rotation angle and it is opposite for left-handed CP (LHCP). An experimental validation of the concept has been recently reported in [12]. The drawback of this concept for discriminating in dual $\mathrm{CP}$ is that there is no possibility for a further phase adjustment since the VRT is used just to generate two symmetrical beams in orthogonal CP. This limitation was overcome in [13], where a flat reflectarray demonstrator generating two focused beams in dual $\mathrm{CP}$ was reported. The reflectarray cell was made of two orthogonal sets of three parallel dipoles, where the lengths are adjusted to focus the beam while the beam splitting is achieved by VRT.

The previous reported works for generation of separate beams in LHCP and RHCP operate in a single frequency. However, multibeam satellite antennas in $K a$-band should operate in two frequency bands, $20 \mathrm{GHz}$ to transmit (Tx) and $30 \mathrm{GHz}$ to receive $(\mathrm{Rx})$. Different reflectarrays have been proposed to operate at multiple frequencies [14]-[18]. A reflectarray to Tx and Rx at both $\mathrm{Ka}$ - and $\mathrm{X}$-bands has been demonstrated [14] by using a separate reflectarray for each band and a frequency selective surface. Other concepts are based on stacked reflectarray layers operating at different frequencies [15]. Several single-layer reflectarrays that operate at $\mathrm{Tx}$ and $\mathrm{Rx}$ in $\mathrm{Ka}$-band have been proposed [16]-[18] using multiresonant elements. None of the previous works allows to implement an independent phase control in each polarization, either because of the symmetry of the printed elements [16] or because the VRT is used to focus the beam in a single CP [17], [18].

In this letter, a reflectarray cell is proposed to provide opposite phase shifts in each orthogonal CP by VRT at two separate frequencies. The cell has been designed and characterized at $\mathrm{Tx}$ and $\mathrm{Rx}$ frequencies for multibeam satellite antennas in $\mathrm{Ka}$ band (19.7 GHz for Tx and $29.5 \mathrm{GHz}$ for Rx). In addition to the application of VRT independently in two frequencies, the cell provides an additional phase adjustment in Rx, which can be used to shape the beam or to compensate geometrical errors, such as the different position of the phase center of the feed at $\mathbf{T x}$ 


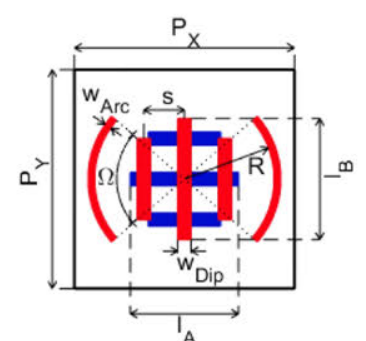

(a)

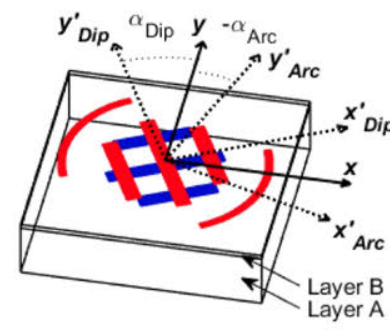

(b)
Fig. 1. Proposed reflectarray cell. (a) Top view with nonrotated elements. (b) Three-dimensional view with rotated elements.

and Rx. The proposed reflectarray cell can be used to design flat reflectarrays with independent beam shaping at two frequencies by applying VRT [9]-[11], and also to design parabolic reflectarrays that generate two adjacent beams per feed in orthogonal $\mathrm{CP}$ in $\mathrm{Tx}$ and $\mathrm{Rx}$.

\section{DEFINITION OF REFLECTARRAY CELL}

\section{A. Operating Principle}

The VRT consists on designing the reflectarray cells to introduce a $180^{\circ}$ phase difference between the two orthogonal linear components of the reflected field when the reflectarray is illuminated by a CP incident field. Under this condition, the rotation of the printed elements in the reflectarray cell by an angle $\alpha_{\text {rot }}$ (see Fig. 1, where $\alpha_{\text {rot }}=\alpha_{\text {Arc }}$ for the Tx elements, and $\alpha_{\text {rot }}$ $=\alpha_{\text {Dip }}$ for the Rx elements) results in a phase shift of $2 \cdot \alpha_{\text {rot }}$ for RHCP and $-2 \cdot \alpha_{\text {rot }}$ for LHCP [10], [2]. This result, originally obtained assuming normal incidence [11], is evaluated here under oblique incidence. Assuming an incident field in $\mathrm{CP}$ $\left(E_{i}^{\mathrm{RHCP} / \mathrm{LHCP}}\right)$ impinging at an oblique angle of incidence on each reflectarray cell, the ideal reflection matrix of the cell to provide a different phase shift in each CP by VRT will be

$$
\left(\begin{array}{l}
E_{r}^{\mathrm{RHCP}} \\
E_{r}^{\mathrm{LHCP}}
\end{array}\right)=\left(\begin{array}{cc}
e^{j \phi_{C P}} & 0 \\
0 & e^{-j \phi_{C P}}
\end{array}\right)\left(\begin{array}{l}
E_{i}^{\mathrm{RHCP}} \\
E_{i}^{\mathrm{LHCP}}
\end{array}\right) e^{j \phi_{0}}
$$

where $\phi_{\mathrm{CP}}$ is the progressive phase required in RHCP, and $\phi_{0}$ is a phase adjustment that can be added by the reflectarray cell to properly shape the beam or to correct phase errors on the reflectarray surface. Several coordinate transformations have been applied to (1): first, from the $\mathrm{CP}$ components to spherical coordinates; second, from spherical coordinates to Cartesian coordinates of the cell [ $x$ and $y$, in Fig. 1(b)]; and finally, the local coordinate system is rotated by an angle $\alpha_{\text {rot }}=\phi_{\mathrm{CP}} / 2\left[x_{\mathrm{Arc}}^{\prime}\right.$, $y_{\text {Arc }}^{\prime}$, for arcs and $x_{\text {Dip }}^{\prime}, y_{\text {Dip }}^{\prime}$, for dipoles, in Fig. 1(b)]. After these transformations, we obtain the ideal reflection matrix, which relates the reflected and incident field components in the locally rotated coordinate system as

$$
\left(\begin{array}{c}
E_{r, x^{\prime}} \\
E_{r, y^{\prime}}
\end{array}\right)=\left(\begin{array}{ll}
R_{x x}^{\prime} & R_{x y}^{\prime} \\
R_{y x}^{\prime} & R_{y y}^{\prime}
\end{array}\right) \cdot\left(\begin{array}{c}
E_{i, x^{\prime}} \\
E_{i, y^{\prime}}
\end{array}\right) e^{j \phi_{0}} .
$$

When computing the coefficients of $\left[\mathbf{R}^{\prime}\right]$ for applying VRT, it has been checked that the conditions to be fulfilled are: $R_{x x}^{\prime}=-R_{y y}^{\prime}$ and $R_{x y}^{\prime}=R_{y x}^{\prime}=0$. Thus, apart from the $180^{\circ}$ phase difference between $R_{x x}^{\prime}$ and $R_{y y}^{\prime}$, the cross-polarization coefficients should be zero. In practice, levels below $-20 \mathrm{~dB}$ provide accurate results for (1).

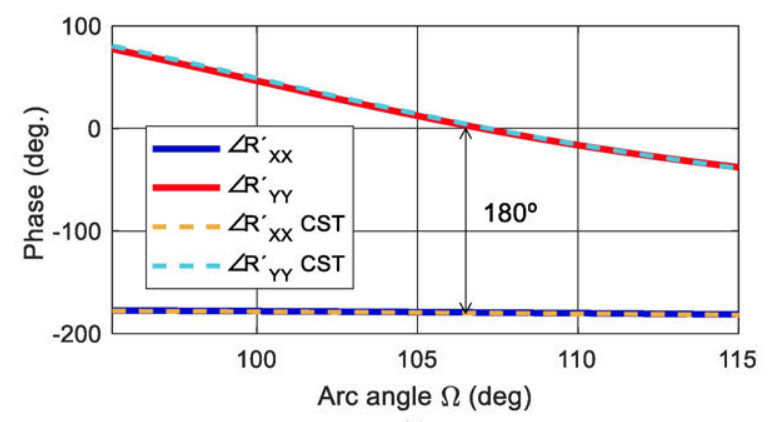

(a)

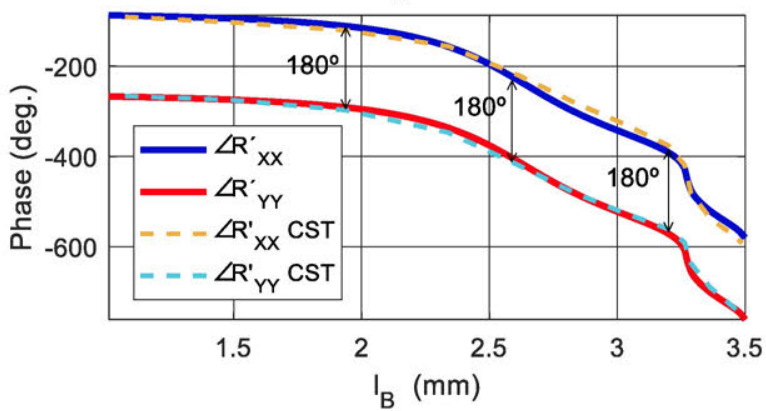

(b)

Fig. 2. Phases of the LP reflection coefficients versus (a) the arc length at 19.7 GHz and (b) the upper central dipole length at $29.5 \mathrm{GHz}$.

\section{B. Definition of the Reflectarray Cell}

The proposed cell combines different types of printed elements: two orthogonal sets of three coupled parallel dipoles printed on the opposite sides of a thin dielectric sheet, and two symmetrical arcs printed on the top surface, as shown in Fig. 1. The inner radius and the width of the ares have been fixed to $R=2.65 \mathrm{~mm}$ and $w_{\text {Arc }}=0.2 \mathrm{~mm}$, respectively. The length of the symmetrical circular arcs is adjusted through its angle $\Omega$ [see Fig. 1(a)] to fulfill the $180^{\circ}$ phase difference between the orthogonal linear components $\left(R_{y y}^{\prime}=-R_{x x}^{\prime}\right)$ at $19.7 \mathrm{GHz}$ [see Fig. 2(a)]. The dipole width is $w_{\text {Dip }}=0.4 \mathrm{~mm}$, and the separation between laterally coupled dipoles is $s=1.2 \mathrm{~mm}$ from center to center. The lengths of the two orthogonal sets of dipoles are used to independently control each linear component of the field at $29.5 \mathrm{GHz}(\mathrm{Rx})$, providing a phase range larger than $360^{\circ}$ [19], while enforcing the condition of $180^{\circ}$ phase shift between the two linear components [see Fig. 2(b)]. Thus, the crossed dipoles are able to introduce a phase adjustment to properly shape the beams at Rx in addition to the phase shift introduced in each $\mathrm{CP}$ by VRT. The lengths of lateral dipoles of each group are scaled by a factor of 0.77 with respect to the lengths of the central dipoles $\left[l_{A}\right.$ and $l_{B}$ in Fig. 1(a)] to ensure linear phase response and a phase range larger than $400^{\circ}$ [see Fig. 2(b)].

The cell has a period of $6.5 \mathrm{~mm} \times 6.5 \mathrm{~mm}$ to avoid the appearance of grating lobes at $29.5 \mathrm{GHz}$ for a maximum angle of incidence of $34^{\circ}$ and to allow enough room for the elements. The dielectric layers are two Diclad 880B substrates (dielectric constant $\left(\varepsilon_{r}\right)=2.17$, loss tangent $\tan \delta=0.0009$ ) with a thickness of $1.524 \mathrm{~mm}$ for the lower layer (A) and $0.127 \mathrm{~mm}$ for the top layer (B). Layer B has been chosen very thin to ensure a similar behavior for the two sets of orthogonal dipoles printed on both sides of the upper dielectric. The $\varepsilon_{r}$ is selected to achieve a low influence of the dipoles in the phase control at $19.7 \mathrm{GHz}$ and to reduce the sensitivity of the condition $R_{x x}^{\prime}=-R_{y y}^{\prime}$ with frequency. 


\section{Cell Performance and Validation}

A homemade analysis routine based on the Method of Moments in the Spectral Domain (SD-MoM), assuming a periodic structure, has been used for the analysis of the cell. This technique has been applied in previous works to analyze other cells based on similar resonant elements, obtaining accurate results [20], [21]. The simulations obtained by the SD-MoM have been validated by the comparison of the results with the commercial software CST Studio Suite [23].

First, the reflection coefficients associated to the orthogonal LP and CP field components have been computed at two frequencies, considering an angle of incidence of $\theta_{i}=13^{\circ}, \phi_{i}=0^{\circ}$. Fig. 2(a) shows the phase of the copolar reflection coefficients for both linear components $\left(R_{X X}^{\prime}\right.$ and $\left.R_{Y Y}^{\prime}\right)$ at $19.7 \mathrm{GHz}$ as the length of the symmetrical arcs is increased. The lengths of the central dipoles on the lower and upper layer are fixed to $l_{A}=2.8 \mathrm{~mm}$ and $l_{B}=2.34 \mathrm{~mm}$, respectively. Note that the lengths of the lateral dipoles on each layer are referred to the lengths of the central ones and the elements have not been rotated yet. The phase curves present a smooth variation, and they are in good agreement with the simulations obtained by CST. The $180^{\circ}$ phase difference between the two linear components that ensures the condition $R_{x x}^{\prime}=-R_{y y}^{\prime}$ is achieved at $\Omega=107^{\circ}$ (an arc length of $5.14 \mathrm{~mm}$ ). Simulated losses are below $0.1 \mathrm{~dB}$.

The phase of the reflection coefficients for both linear components at $29.5 \mathrm{GHz}$ is presented in Fig. 2(b) as the lengths of the dipoles are increased. The graphic only indicates the length of the central dipole on the upper layer, $l_{B}$, since the length of the central dipole on the lower layer is adjusted between $l_{A}=2.5 \mathrm{~mm}$ and $5 \mathrm{~mm}$ to provide the required $180^{\circ}$ phase difference to ensure $R_{x x}^{\prime}=-R_{y y}^{\prime}$. The length of the arcs was fixed to $5.14 \mathrm{~mm}\left(\Omega=107^{\circ}\right)$, assuming nonrotated elements. The phases present a smooth variation within a range larger than $400^{\circ}$ where the $180^{\circ}$ phase difference is fulfilled, which enables to adjust the lengths of the dipoles on one layer to provide the phase shift required for the phase correction at $29.5 \mathrm{GHz}$, whereas the orthogonal dipoles on the other layer are adjusted to fulfill the $180^{\circ}$ phase difference. Simulated losses are below $0.5 \mathrm{~dB}$.

The phase of the copolar reflection coefficients for the orthogonal CP components has been computed at both $\mathrm{Tx}$ and Rx frequencies (19.7 and $29.5 \mathrm{GHz}$ ) when the appropriate elements are rotated counterclockwise (arcs for Tx, dipoles for $\mathrm{Rx}$ ), while the other elements remain unrotated (dipoles for Tx, arcs for Rx); see Fig. 3. The results are shown as a function of the rotation angle: $\alpha_{\text {Arc }}$ for Tx and $\alpha_{\text {Dip }}$ for Rx. The phases have been referenced to the phase of $R_{X X}^{\prime}$, so that the value of the RHCP and LHCP phases at the cross point, $\alpha_{\text {rot }}=0^{\circ}$, is equal to $0^{\circ}$. First, the appropriate elements [the arcs in Fig. 3(a), the dipoles in Fig. 3(b)] have been rotated without adjusting their dimensions ("Fixed dim.," plotted in solid lines in Fig. 3). Note that the reflectarray elements had been previously designed to provide the $180^{\circ}$ phase difference with nonrotated elements $\left(\alpha_{\text {rot }}=0^{\circ}\right)$ : The arc length corresponds to $\Omega=107^{\circ}$, and the dipole lengths are $l_{A}=2.8 \mathrm{~mm}$ and $l_{B}=2.35 \mathrm{~mm}$. The phases obtained by SD-MoM are virtually identical to those obtained by CST (marked with crosses in Fig. 3), validating the analysis tool for both frequency bands. Ideally, the CP phases should be proportional to twice the rotation angle of the elements $\left( \pm 2 \cdot \alpha_{\text {rot }}\right.$, plotted in dotted lines in Fig. 3$)$, but there are some errors, which are up to $40^{\circ}$ at $29.5 \mathrm{GHz}$ [see Fig. 3(b)]. To reduce the phase errors, the rotated elements are adjusted for each rotation angle to fulfil the $180^{\circ}$ phase difference, and the results ("Opt. dim.," in dashed lines in Fig. 3) show a better

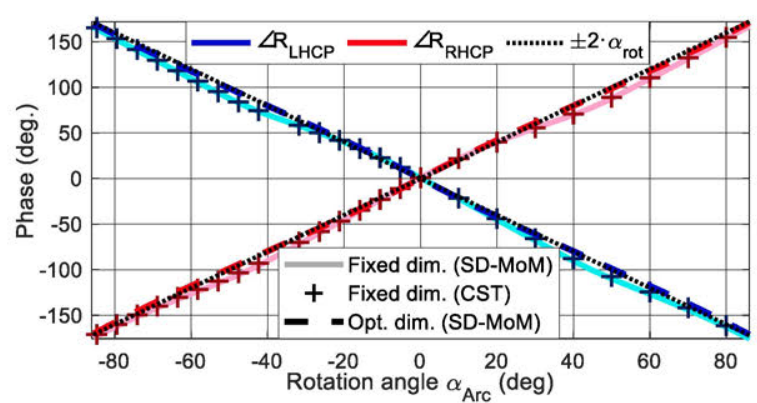

(a)

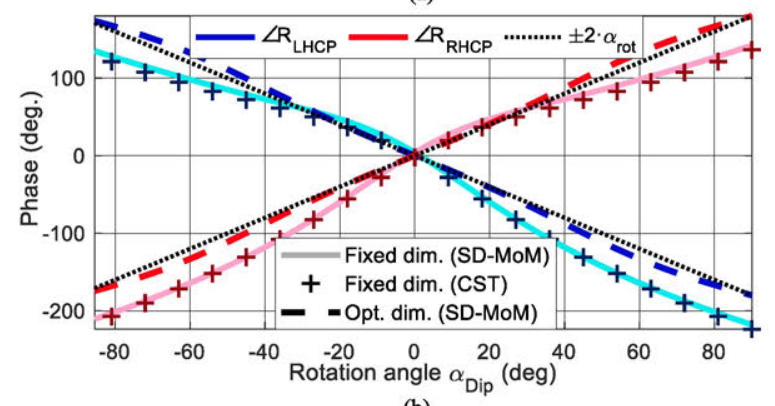

(b)

Fig. 3. Phases of the $\mathrm{CP}$ reflection coefficients (a) at $19.7 \mathrm{GHz}$ versus arc rotation angle and (b) at $29.5 \mathrm{GHz}$ versus dipole rotation angle before and after adjusting the dimensions of the elements for each rotation angle.

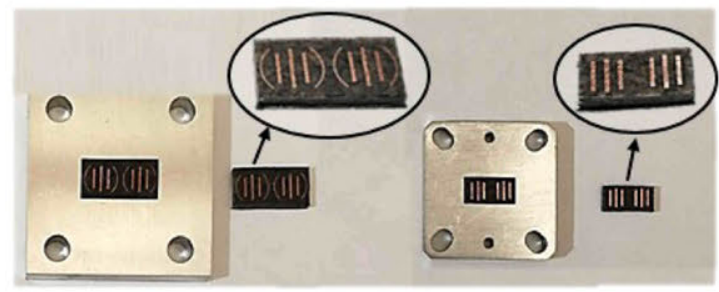

Fig. 4. Setup for measuring samples in waveguide simulator.

concordance with the ideal response (the largest difference is $13^{\circ}$, which appears at $29.5 \mathrm{GHz}$ for $\pm 60^{\circ}$ rotation). Since the phase difference between the LP components at $29.5 \mathrm{GHz}$ has been enforced to $180^{\circ}$, the phase error in CP is produced by an increase of the cross-polar linear components $\left(R_{X Y}^{\prime}\right.$ and $\left.R_{Y X}^{\prime}\right)$. The simulated level of losses in CP (not shown here) is below $0.2 \mathrm{~dB}$ at $19.7 \mathrm{GHz}$. At $29.5 \mathrm{GHz}$, the losses are worse than $1 \mathrm{~dB}$ for $\alpha_{\text {Dip }}>50^{\circ}$ when the rotated dipoles are kept fixed, and they have been reduced to $<0.5 \mathrm{~dB}$ after the adjustment for each rotation angle. The reduction in $\left|R_{X X}^{\prime}\right|$ and $\left|R_{Y Y}^{\prime}\right|$ at $29.5 \mathrm{GHz}$ for large rotation angles is due to an increase of the cross-polar components, which leads to higher phase errors, since the condition $R_{X Y}^{\prime}=R_{Y X}^{\prime}=0$ is not accurately accomplished.

\section{Measurements of Cells in Waveguide Simulator}

Several samples formed by two reflectarray cells have been manufactured and measured using the well-known waveguide simulator technique WGS (see Fig. 4) to test the performance of the reflectarray cell in a periodic environment [22]. Since the analysis tool based on SD-MoM has been already validated by comparison with simulations from CST, the results obtained here are used to accurately characterize the electrical properties of the materials, which may show some variations with respect to the nominal values provided by the manufacturer at $10 \mathrm{GHz}$. The dielectric employed for the WGS samples is IsoClad 917 


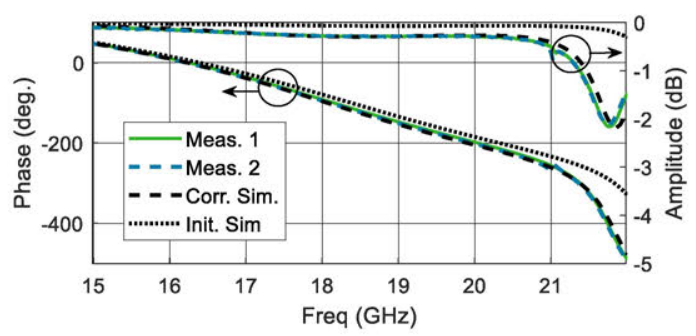

Fig. 5. Reflection coefficient in module and phase of the reflectarray cells placed in a WR51 waveguide.

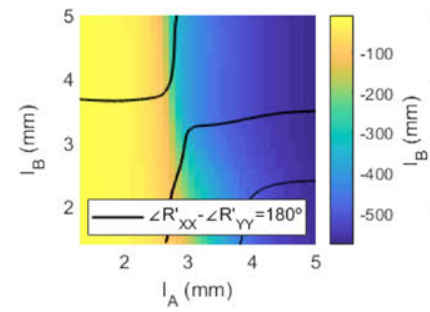

(a)

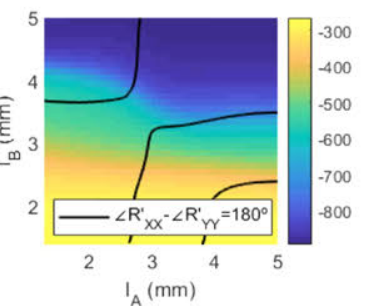

(b)
Fig. 6. Phases of the cell reflection coefficients versus dipole lengths for (a) $X$ - and (b) $Y$-pol. at $29.5 \mathrm{GHz}$.

with nominal values: $\varepsilon_{r}=2.17, \tan \delta=0.0013$ and thickness of $1.524 \mathrm{~mm}$. The waveguide allows to analyze the cell response associated to vertical polarization, so there is no necessity to add horizontal dipoles, and hence, only one layer of substrate is used. The samples are placed in WR51 and WR34 waveguides (dimensions $12.95 \mathrm{~mm} \times 6.48 \mathrm{~mm}$ and $8.64 \mathrm{~mm} \times 4.32 \mathrm{~mm}$, respectively) to analyze the response within the frequency band of each waveguide (15-22 GHz and 22-33 GHz, respectively). The lengths of the printed arcs and dipoles for the WR51 samples are $\Omega=110.4^{\circ}$ and $l_{B}=4.2 \mathrm{~mm}$. The samples in WR34 waveguide only include three dipoles $\left(l_{B}=3.1 \mathrm{~mm}\right)$ and are used to characterize the material at higher frequencies.

The measured curves of amplitude and phase of the reflection coefficient for two manufactured samples in $K$ band are shown in Fig. 5 with the simulations, considering the variation of the angle of incidence with frequency into the waveguide. Note that the measurements of the two samples are virtually identical, demonstrating a good repeatability. The dielectric properties of the substrate have been slightly adjusted to match simulations and measurements since simulations were already validated with CST. The following values are obtained: $\varepsilon_{r}=2.32, \tan \delta=0.0052$ for the frequencies associated to the WR51, and $\varepsilon_{r}=2.3, \tan \delta=$ 0.0058 within the frequency band of the WR34. Since the values obtained at both frequency bands are very similar, the following values have been used to repeat the simulations in Fig. 5 ("Corr. Sim."): $\varepsilon_{r}=2.3, \tan \delta=0.005$. Although the loss tangent has been multiplied by a factor of 5 , the measured losses are still lower than $0.5 \mathrm{~dB}$ in practically the whole frequency band, except at resonance, where the losses may increase up to $2 \mathrm{~dB}$. Note that in a real reflectarray just a few cells will be on resonance, and the average losses are expected to be less than $0.5 \mathrm{~dB}$.

\section{PARAMETRIC StUdy OF REFLECTARRAY CELL}

Once the SD-MoM analysis software has been validated and the dielectric material accurately characterized, a parametric study of the reflectarray cell has been carried out in order to test the performance of the cell. Fig. 6 shows the phase of the copolar LP reflection coefficients $\left(R_{X X}\right.$ and $\left.R_{Y Y}\right)$ at $29.5 \mathrm{GHz}$

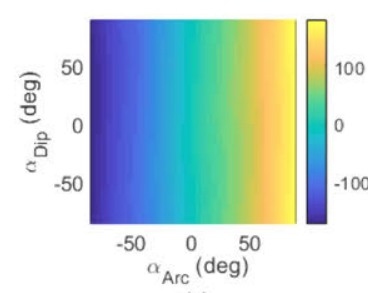

(a)

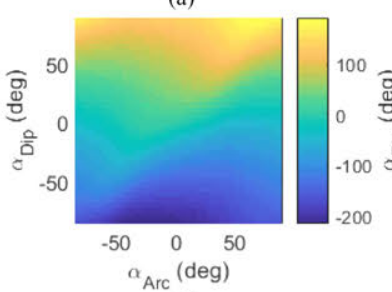

(c)

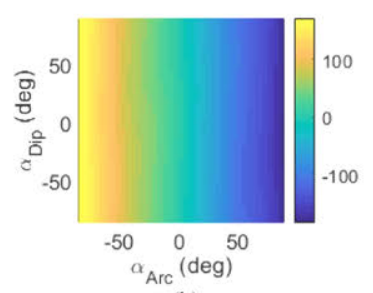

(b)

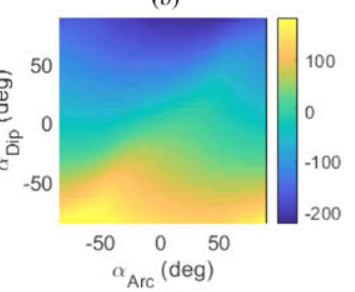

(d)
Fig. 7. Phase of the cell reflection coefficients versus rotation angle of the elements for (a) RHCP and (b) LHCP at $19.7 \mathrm{GHz}$, and (c) RHCP and (d) LHCP at $29.5 \mathrm{GHz}$.

with respect to the lengths of the central dipoles on both layers, considering nonrotated elements and arcs defined by $\Omega=107^{\circ}$. This figure shows that the orthogonal dipoles on each layer are practically uncoupled, so each LP can be independently controlled by the length of the appropriate dipoles: The dipoles on layer A are oriented in the direction of $x$-axis, so the phase in $X$-pol. can be mostly controlled by these dipoles [see Fig. 6(a)]. Similarly, the phase in $Y$-pol. can be adjusted by varying the dipole lengths in layer $\mathrm{B}$, oriented in the direction of $y$-axis [see Fig. 6(b)]. The figures include the lines where the phase difference between both polarizations is $180^{\circ}$. Note that both polarizations present a phase range larger than $400^{\circ}$, as intended. Simulated losses are below $0.5 \mathrm{~dB}$.

Fig. 7 shows the phase response of the cell in both CP components at 19.7 and $29.5 \mathrm{GHz}$, when arcs and dipoles are independently rotated, considering fixed dimensions for the printed elements $\left(\Omega=107^{\circ}, l_{A}=2.8 \mathrm{~mm}\right.$, and $\left.l_{B}=2.34 \mathrm{~mm}\right)$. As can be seen in Fig. 7(a) and (b), the phase response at $19.7 \mathrm{GHz}$ can be controlled by the arc rotation angle, achieving an independent operation of the Tx resonant elements with respect to the $\mathrm{Rx}$ elements. This independence between the rotation of arcs and dipoles slightly decreases if longer dipoles are considered. At $29.5 \mathrm{GHz}$, the phase is influenced by $\alpha_{\text {Arc }}$, which indicates the importance of considering the real rotation angles of the arcs in the process of adjusting the crossed dipoles.

\section{CONCLUSION}

A new reflectarray cell has been proposed to provide opposite phase shifts between orthogonal CP at 19.7 and $29.5 \mathrm{GHz}$. The reflectarray cell allows to implement the VRT with a dualfrequency operation, through independent rotation of arcs and dipoles. The proposed reflectarray cells are presently being used to design a $1.8 \mathrm{~m}$ parabolic reflectarray to generate two $0.65^{\circ}$ wide beams deviated $0.28^{\circ}$ in RHCP and $-0.28^{\circ}$ in LHCP at Tx and $\mathrm{Rx}$, when illuminated by a dual $\mathrm{CP}$ feed. The preliminary results show a minimum a edge-of-coverage gain around $45 \mathrm{dBi}$ and sidelobes that produce a carrier-to-interference ratio close to $20 \mathrm{~dB}$ at 19.7 and $29.5 \mathrm{GHz}$. According to the preliminary results, the proposed reflectarray cells could allow a reduction in the number of feeds and antennas required in multispot satellites, compared with conventional reflectors. 


\section{REFERENCES}

[1] M. Schneider, C. Hartwanger, and H. Wolf, "Antennas for multiple spot beams satellites,” CEAS Space J., vol. 2, pp. 59-66, Dec. 2011.

[2] M. Zhou and S. B. Sørensen, "Multi-spot beam reflectarrays for satellite telecommunication applications in Ka-band," in Proc. 10th Eur. Conf. Antennas Propag., Davos, Switzerland, 2016, pp. 1-5.

[3] D. Martinez-de-Rioja, E. Martinez-de-Rioja, and J. A. Encinar, "Multibeam reflectarray for transmit satellite antennas in $\mathrm{Ka}$ band using beamsquint," in Proc. IEEE Int. Symp. Antennas Propag., Fajardo, PR, USA, Jul. 2016, pp. 1421-1422.

[4] J. A. Encinar et al., "Dual-polarization dual-coverage reflectarray for space applications," IEEE Trans. Antennas Propag., vol. 54, no. 10, pp. 28272837, Oct. 2006.

[5] S. R. Rengarajan, "Reflectarrays of rectangular microstrip patches for dualpolarization dual-beam radar interferometers," Prog. Electromagn. Res., vol. 133, pp. 1-15, 2013.

[6] E. Martinez-de-Rioja, J. A. Encinar, R. Florencio, and R. R. Boix, "Reflectarray in $\mathrm{K}$ and Ka bands with independent beams in each polarization," in Proc. IEEE Int. Symp. Antennas Propag., Fajardo, PR, USA, 2016, pp. 1199-1150.

[7] S. Mener, R. Gillard, R. Sauleau, A. Bellion, and P. Potier, "Dua1circularly polarized reflectarray with independent control of polarizations," IEEE Trans. Antennas Propag., vol. 63, no. 4, pp. 1877-1881, Apr. 2015.

[8] M. Hosseini and S. V. Hum, "A dual-CP reflectarray unit cell for realizing independently controlled beams for space applications," in Proc. 11th Eur. Conf. Antennas Propag., Paris, France, Apr. 2017, pp. 66-70.

[9] J. Huang and R. J. Pogorzelski, "A Ka-band microstrip reflectarray with elements having variable rotation angles," IEEE Trans. Antennas Propag., vol. 46, no. 5, pp. 650-656, May 1998.

[10] A. E. Martynyuk, J. I. M. Lopez, and N. A. Martynyuk, "Spiraphase-type reflectarrays based on loaded ring slot resonators," IEEE Trans. Antennas Propag., vol. 52, no. 1, pp. 142-153, Jan. 2004.

[11] J. J. H. Wang, "Characteristics of a new class of diode-switched integrated antenna phase shifter," IEEE Trans. Antennas Propag., vol. AP-31, no. 1, pp. 156-159, Jan. 1983.

[12] A. Somolinos, R. Florencio, I. González, J. A. Encinar, and F. Cátedra, "Experimental validation of generating two spaced beams with reflectarrays by VRT," IEEE Trans. Antennas Propag., vol. 67, no. 6, pp. 4263-4268, Jun. 2019.
[13] R. Florencio, J. A. Encinar, R. R. Boix, M. Barba, and G. Toso, "Flat reflectarray that generates adjacent beams by discriminating in dual circular polarization," IEEE Trans. Antennas Propag., vol. 67, no. 6, pp. 37333742, Jun. 2019

[14] M. Chaharmir and J. Shaker, "Design of a multilayer X-/Ka-band frequency-selective surface-backed reflectarray for satellite applications," IEEE Trans. Antennas Propag., vol. 63, no. 4, pp. 1255-1262, Apr. 2015.

[15] E. Martinez-de-Rioja, J. A. Encinar, M. Barba, R. Florencio, R. R. Boix, and V. Losada, "Dual polarized reflectarray transmit antenna for operation in Ku- and Ka-bands with independent feeds," IEEE Trans. Antennas Propag., vol. 65, no. 6, pp. 3241-3246, Jun. 2017.

[16] M. Abdollahvand, J. A. Encinar, K. Forooraghi, Z. Atlasbaf, and M. Barba, "Single-layer dual-frequency reflectarray for Ka-band antennas," in Proc 10th Eur. Conf. Antennas Propag., Davos, Switzerland, 2016, pp. 1-4.

[17] T. Smith, U. Gothelf, O. S. Kim, and O. Breinbjerg, "An FSS-backed 20/30 GHz circularly polarized reflectarray for a shared aperture L-and Kaband satellite communication antenna," IEEE Trans. Antennas Propag., vol. 62, no. 2, pp. 661-668, Feb. 2014.

[18] T. Smith, U. Gothelf, O. S. Kim, and O. Breinbjerg, "Design, manufacturing, and testing of a $20 / 30 \mathrm{GHz}$ dual-band circularly polarized reflectarray antenna," IEEE Antennas Wireless Propag. Lett., vol. 12, pp. 1480-1483, 2013.

[19] R. Florencio, J. A. Encinar, R. R. Boix, and G. Perez-Palomino, "Dual polarisation reflectarray made of cells with two orthogonal sets of parallel dipoles for bandwidth and cross-polarisation improvement," Microw., Antennas Propag., vol. 8, no. 15, pp. 1389-1397, 2014.

[20] R. Florencio, R. R. Boix, E. Carrasco, J. A. Encinar, and V. Losada "Efficient numerical tool for the analysis and design of reflectarrays based on cells with three parallel dipoles," Microw. Opt. Technol. Lett., vol. 55, no. 6, pp. 1212-1216, Jun. 2013.

[21] R. Florencio, R. R. Boix, and J. A. Encinar, "Efficient spectral domain MoM for the design of circularly polarized reflectarray antennas made of split rings," IEEE Trans. Antennas Propag., vol. 67, no. 3, pp. 1760-1771, Mar. 2018.

[22] P. W. Hannan and M. A. Balfour, "Simulation of a phased-array antenna in waveguide," IEEE Trans. Antennas Propag., vol. AP-13, no. 3, pp. 342353, May 1965.

[23] CST Microwave Studio, Computer Simulation Technology. [Online]. Available: www.cst.com, Accessed on: July 03, 2019. 